\title{
ON EVALUATION OF MOTION GAITS ENERGY EFFICIENCY WITH A HEXAPOD CRAWLING ROBOT
}

\author{
LukÁŠ ČernÝ*, Petr ČížEK, JAN FAIGL \\ Czech Technical University, Faculty of Electrical Engineering, Technicka 2, Prague, Czech Republic \\ * corresponding author: cernylu6@fel.cvut.cz
}

\begin{abstract}
In this work, we are concerning the problem of energy efficient locomotion of a hexapod crawling robot. We are emphasizing a practical verification and deployment on a real walking robot to evaluate relations between the energy consumption, motion speed, and terrain type with a particular motion gait. The tripod, tetrapod, and pentapod motion gaits are considered in the presented evaluation report.
\end{abstract}

KEYWORDS: RGB-D SLAM, localization, ground-truth construction, legged robot, rough terrain traversal, adaptive motion gait.

\section{INTRODUCTION}

Energy efficient and fast locomotion is a key ability for the mobile robot to extent its operation capabilities. As legged robots offer the potential to traverse various types of terrains, it is desirable to adjust their locomotion to be as energy efficient as possible. In general, there are two principal ways of locomotion control for legged robots operating in rough terrains. Either deliberative control where individual foot-tip positions of each leg are planned in advance (e.g., [1]) or reactive control based on a repetitive motion gait with prescribed leg trajectories with a certain amount of adaptability such as 2. The later approach builds on the fact that legged robots often utilize collections of gait patterns to locomote over a variety of surfaces with each gait tuned for a specific surface and set of operational conditions. Several commonly used gait patterns such as tripod, tetrapod, and pentapod motion gaits have been developed for hexapod crawling robots.

During traversing a rough terrain, it is possible to select a particular gait pattern and stick with it for the whole process of the navigation; however, it may be beneficial to utilize knowledge of the terrain currently being traversed to select the most appropriate gait to optimize a particular criterion, e.g., speed, stability, or energy consumption.

The gait selection can be based on both exteroceptive and proprioceptive sensing. In this paper, we consider proprioceptive sensory data only, specifically the current sensory data to evaluate the energy efficiency of individual gaits on different terrain types. We use the information about the overall current drawn from the robot energy source (the used battery pack) to compute instantaneous power and consequently the consumed energy during the robot operation.

We performed a series of experiments to collate information about the energy efficiency of different gaits on different terrain types to establish a gait selection strategy that would let us alter gaits according to the terrain that is being traversed. For this purpose, we expect the terrain to be foreknown, i.e., classified in advance. We distinguish following terrain types: office floor, grass, inclined plane, and wooden blocks, see Figure 1. Online terrain classification can be done using exteroceptive data; however, this topic is out of the scope of this paper, therefore in the herein presented evaluation of the energy consumption, we rather assume the current terrain type is available.

An evaluation of the energy efficiency applied for the hexapod crawling robot has been shown in [3]. The authors propose a method of energetic cost evaluation and they applied it in a gait transition algorithm that alters between two different gaits according to the power consumption and thus, according to the particular terrain type. Several methods of traversability cost evaluation based on proprioceptive sensors are proposed in 4]. The authors also consider a hexapod walking robot and utilize information about the current torque at the leg joints to estimate the power consumption. Contrary to this approach, we consider a direct current measurement in this paper, which provides a more precise estimation of the overall power consumption during crawling.

This paper is organized as follows. Section 2 describes the utilized hexapod robot platform, the considered motion gait patterns, and used evaluation methodology for measuring the energy efficiency of the individual motion gaits. Section 3 is dedicated to the experimental results. Finally, in Section 4 , we discuss the experimental results and propose further research directions.

\section{Problem Statement}

\subsection{HEXAPOD PLATFORM}

The idea of optimizing the energy consumption by altering gaits according to the terrain type can be applied to any legged robot. In this work, we utilize a robot based on an off-the-shelf Phantom X Mark 


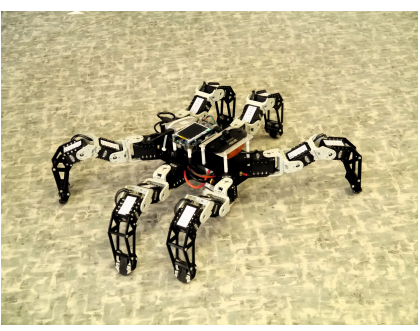

(A) . Office floor

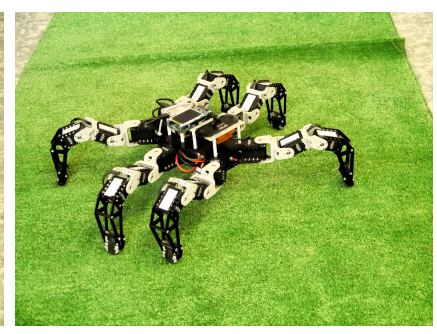

(в) . Grass

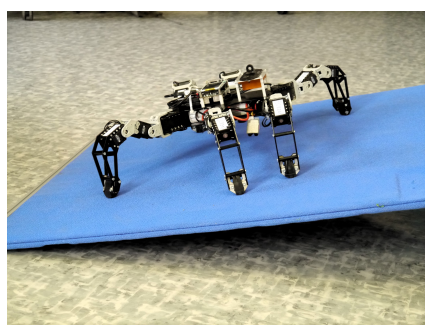

(c) . Inclined plane

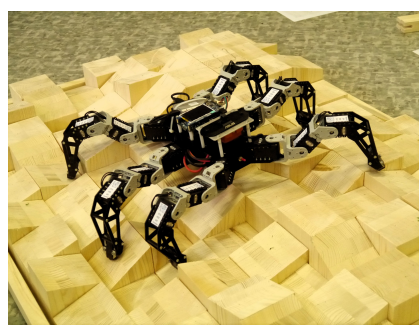

(D) . Wooden blocks

Figure 1. Terrain types.

II platform with six legs. Each leg consists of three actuated joints driven by the Dynamixel AX-12 intelligent servos. This makes the robot easy to use and relatively cheap with surprisingly large locomotion capabilities in rough environments.

\subsection{Motion Gaits}

A straightforward approach to a hexapod robot locomotion is to predefine and fix a particular motion gait pattern and simply repeat such a pattern over and over. Based on the number of legs moving simultaneously and on the order in which the legs are altering between the support phase and the swing phase, different motion gaits can be recognized. This include regular tripod, tetrapod, pentapod, and ripple gaits. Further details on these regular gaits can be found in [5].

A basic locomotion controller assume crawling on a flat terrain by default; however, it is necessary to negotiate the landscape using the robot sensory feedback to surpass a rough terrain. In this report, we consider the default tripod, tetrapod, pentapod, and ripple gaits which assume crawling on a flat terrain and their adaptive variants for tripod, tetrapod, and pentapod gaits [2].

The adaptive gaits exploit the sensory feedback from the intelligent servo drives to detect the contact point of the leg with the supporting surface [6. In particular, a position error of the servo joint, which is proportional to the applied torque, is utilized to stop the motion of the leg during the swing phase, and thus to detect the contact point of the leg with the ground.

\subsection{ENERGETICS}

The fundamental sensory input for the proposed evaluation of the energy efficiency of the individual gaits is based on measuring of the current $I$ drawn from the robot main battery. From the measured current $I$ we can compute the instantaneous power as

$$
P_{\text {in }}=V I,
$$

where $I$ is the instantaneous current (in units of Amperes), $V$ is voltage of the battery (in units of Volts), and $P_{\text {in }}$ is the instantaneous power (in units of Watts). As shown in [3], $P_{\text {in }}$ is the total power consumed by the robot that consists of the power generating mechanical forces and the power representing the energy loss. However, we consider the total power only because we aim to minimize the overall energy consumed.

From the gathered information about the instantaneous power (1), we can estimate the energy consumed during traversing a particular terrain. Based on the relationship between the consumed energy $E$ and the instantaneous power

$$
P_{\text {in }}(t)=\frac{\mathrm{d} E(t)}{\mathrm{dt}}
$$

we can write

$$
E(t)=\int_{0}^{t} P_{\text {in }}(\tau) \mathrm{d} \tau,
$$

where $E(t)$ is the energy consumed (in units of Joules) from the beginning of the experiment up until the specified time $t$. From (3) we can estimate the overall energy consumption by integrating the instantaneous power numerically, e.g., using the trapezoidal rule.

\section{Evaluation}

\subsection{EXPERIMENTAL SET-UP}

The following experimental setup has been designed to evaluate and compare particular abilities of each considered gait to traverse a particular terrain type. The robot has been requested to walk a fixed distance of one meter straight ahead and traverse a particular terrain type. Considered terrain types are the office floor, artificial grass, inclined plane, and wooden blocks of irregular heights (see Figure 1). The office floor and artificial grass represent terrain types with different adhesion, and thus with a different slippage. The inclined plane and wooden blocks represent an environment with elevated demands on the power consumption. The locomotion of the robot on the wooden blocks is also often subjected to slippage and abrupt motions which apply further load on the servo drives, leading to a higher energy consumption.

The experiment has been repeated three times for each gait on each terrain type except the default gaits and wooden blocks because the default gaits are not capable of traversing such a rough terrain. The distance traveled by the robot has been estimated based on the legged odometry only. 


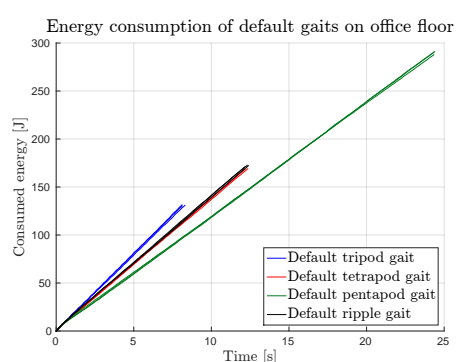

(A) . Default gaits on office floor

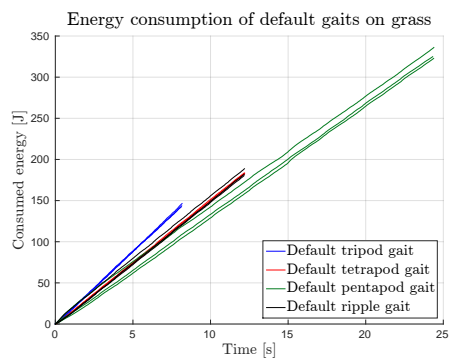

(в) . Default gaits on grass

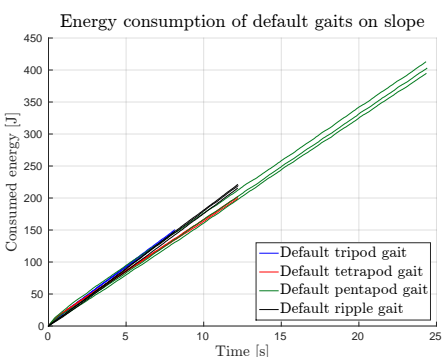

(c) . Default gaits on inclined plane

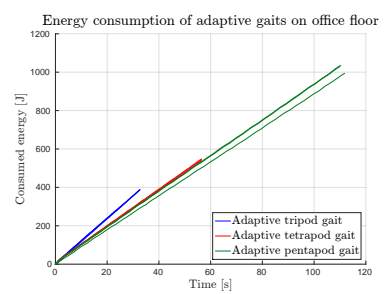

(D) . Adaptive gaits on office floor

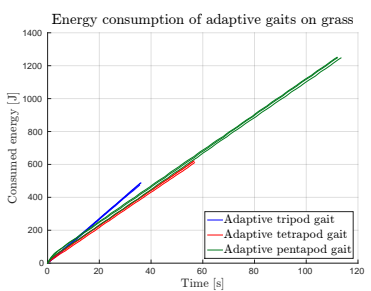

(E) . Adaptive gaits on grass

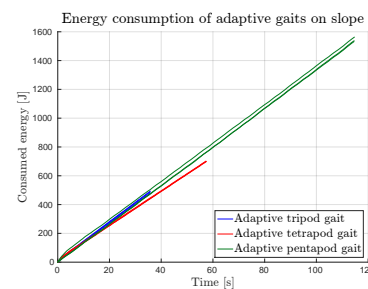

(F) . Adaptive gaits on inclined plane

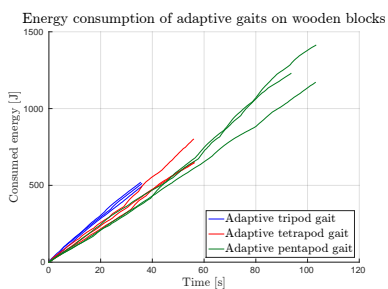

(G) . Adaptive gaits on wooden blocks

FiguRE 2. Energy consumption over time.

The overall current drawn from the battery has been measured using the ACS712 hall effect-based linear current sensor with the measuring range of $\pm 5 \mathrm{~A}$. Each current data sample was provided with a timestamp. The reading frequency was not completely invariant due to the time consumed by the motion control and other factors. In spite of that the reading frequency was approximately $100 \mathrm{~Hz}$.

Before each experiment, we measured the battery voltage and we assumed it is constant during the robot movement because the drop of the voltage was insignificant during the experiment. Thereby, we have acquired all the information needed to estimate the energy consumed by the robot motion.

\subsection{Results}

Having done all the experiments described above, the instantaneous power for each current sample using (1) has been computed. Consequently, the power has been numerically integrated according to (3). The plotted energy consumption over time is presented in Figure2. where the particular plots are as follows. Figure 2a and Figure 2d show the results of the experiments performed on the office floor. Figure 2e and 2e show the results for the artificial grass. Experimental results performed on the inclined plane are shown in Figure 2c and Figure 2f Finally, the results from traversing the wooden blocks are presented in Figure $2 \mathrm{~g}$. The experiments have been run three times for each terrain type and gait. Therefore, results from the particular trials have been grouped and marked by a specific color in the plots. Each color represents one gait pattern, and thus each color group consists of three lines that correspond to the three experiments performed for every gait and terrain type.

\subsection{Discussion}

In Figure 2, we can clearly see that a gait selection can significantly influence the energy efficiency of the robot motion. Tables 1 and 2 and Figure 3 together with Figure 4 summarize the overall energy consumption and the time needed for traversing the terrain (a distance of $1 \mathrm{~m}$ ) by each motion gait. The results were computed as the mean values of the data resulting from each triplet of the performed experiments.

According to Table 1 we can establish a strategy for gait selection to prefer the default tripod gait whenever the particular terrain type can be traversed by it. In rough terrains, the adaptive tripod gait is preferred. Considering the time in Table 1 , the switching strategy that minimizes the time consumption (the speed of the robot) yields the same result.

However, such a gait selection rule optimizes only the energy and time consumption. Different optimization criteria might be induced by the application scenario, e.g., the tripod gait is the least safe gait in terms of the robot stability, and thus it is not capable of traversing such challenging terrains as the pentapod gait, which is the most stable gait.

The results presented above indicate the influence of the energy consumption is proportional to the time by a multiplication factor. The factor can be utilized as a single parameter describing the energy efficiency of the gait pattern on a particular terrain type. The multiplication factor actually coincides with the average power consumed by the robot. Moreover, the average power might be used as a characteristic for the terrain classification. However, to get relevant information about an average power, we need to collect data over a longer time interval because the instantaneous power changes signif- 


\begin{tabular}{lrrrc}
\hline \multirow{2}{*}{ Gait } & \multicolumn{4}{c}{ Consumed energy [J] } \\
\cline { 2 - 5 } & Floor & Grass & Plane & Blocks \\
\hline Def. tripod & 130.9 & 144.0 & 148.3 & - \\
Def. tetrapod & 168.7 & 183.0 & 200.9 & - \\
Def. pentapod & 289.7 & 327.9 & 403.2 & - \\
Def. ripple & 171.9 & 183.7 & 218.4 & - \\
Ad. tripod & 386.6 & 482.5 & 485.6 & 507.6 \\
Ad. tetrapod & 543.1 & 617.0 & 699.0 & 700.0 \\
Ad. pentapod & 1019.1 & 1248.7 & 1543.4 & 1270.3 \\
\hline
\end{tabular}

TABLE 1. Energy consumption.

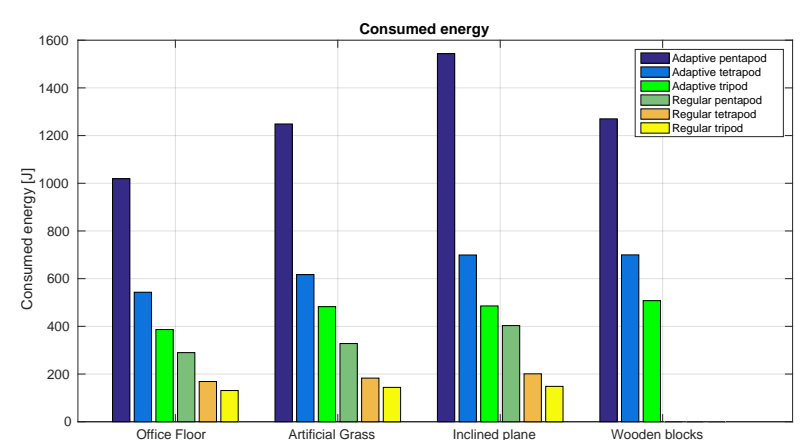

FiguRE 3. Energy consumption on different terrain types.

icantly during a single gait cycle. This can be observed for the terrain of wooden blocks as fluctuations of otherwise relatively straight lines in Figure 2. Based on the presented results, we can deduce that the time interval of few seconds (or few gait cycles) should be sufficient to provide the relevant data.

Notice, the wooden blocks are not, unlike the other terrain types, uniform, and therefore, fluctuations in the estimated energy consumption may be observed in the presented results. The energetic cost together with the traversability of the terrain vary from place to place and they both depend on exact location of the robot in the environment. This observation motivate us that a terrain classification might divide the area into smaller parts and evaluate traversability in these local areas.

Notice that all the gait parameters such as speed, stride length, etc. were fixed for each gait individually for all experiments reported in this paper. However, the energy efficiency can be used in the optimization of the gait and it can be also improved by a mechanical construction of the robot.

A further experimental evaluation can also be enhanced by a precise measurement of the distance traveled by the robot. In this manner, factors such as traction and leg slippages can be taken into account, which may provide additional information about suitability of particular gaits for specific terrain types.

\begin{tabular}{lrrrl}
\hline Gait & \multicolumn{4}{c}{ Time [s] } \\
\cline { 2 - 5 } & Floor & Grass & Plane & Blocks \\
\hline Def. tripod & 8.1 & 8.1 & 8.1 & - \\
Def. tetrapod & 12.2 & 12.2 & 12.2 & - \\
Def. pentapod & 24.4 & 24.4 & 24.3 & - \\
Def. ripple & 12.3 & 12.2 & 12.2 & - \\
Ad. tripod & 32.6 & 35.8 & 35.7 & 35.7 \\
Ad. tetrapod & 56.5 & 56.6 & 57.3 & 56.1 \\
Ad. pentapod & 110.6 & 112.5 & 114.6 & 95.8 \\
\hline
\end{tabular}

TABLE 2. Traversing time.

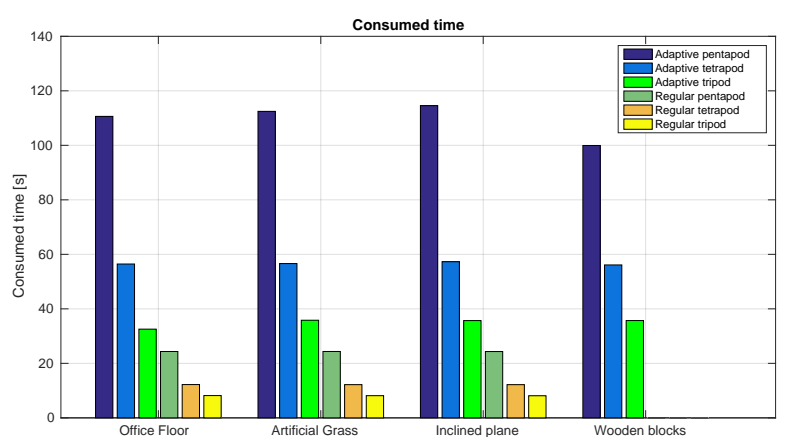

Figure 4. Traversing time on different terrain types.

\section{Conclusion}

In this work, we study the problem of energetic characteristics of the hexapod legged robot locomotion. We have evaluated the consumed energy for the most common motion gait types and different terrain types. From the presented experimental results, we can observe that the default tripod gait is the fastest and the most energy efficient gait for flat terrains. This observation yields the main strategy for the gait switching rule that minimizes the overall energy consumption. The gait switching rule can be formulated such as whenever the default gait is not capable of traverse a terrain due to the terrain roughness, its adaptive version should be preferred.

The results indicate the proportional relation between the operational time and the energy consumption, which, in fact, is not a surprising result. However, the multiplication factor can be used as a characteristic for the terrain classification.

All the proposed applications and implications of presented results are intended as a motivation for further research. A deployment of the gait switching decision rule and further investigation of the gait optimal parametrization using the energy characteristics evaluation is of our particular interest.

\section{ACKNOWLEDGEMENTS}

The presented work was supported by the Czech Science Foundation (GAČR) under research project No. 15-09600Y. The support of grant No. SGS16/235/OHK3/3T/13 to Petr Čížek is also gratefully acknowledged. 


\section{REFERENCES}

[1] D. Belter, P. Skrzypczynski. Rough terrain mapping and classification for foothold selection in a walking robot. Journal of Field Robotics 28(4):497-528, 2011. DOI:10.1002/rob.20397.

[2] J. Mrva, J. Faigl. Tactile sensing with servo drives feedback only for blind hexapod walking robot. In Robot Motion and Control (RoMoCo), 2015 10th International Workshop on, pp. 240-245. 2015. DOI:10.1109/RoMoCo.2015.7219742.

[3] N. Kottege, C. Parkinson, P. Moghadam, et al. Energetics-informed hexapod gait transitions across terrains. In IEEE International Conference on Robotics and Automation (ICRA), pp. 5140-5147. 2015. DOI:10.1109/ICRA.2015.7139915
[4] J. Mrva, M. Stejskal, J. Faigl. On traversability cost evaluation from proprioceptive sensing for a crawling robot. In Acta Polytechnica CTU Proceedings, pp. 34-39. 2015. DOI:10.14311/APP.2015.1.0034

[5] G. Dudek, M. Jenkin. Computational Principles of Mobile Robotics. Cambridge University Press, New York, NY, USA, 2000.

[6] J. Mrva, J. Faigl. Tactile sensing with servo drives feedback only for blind hexapod walking robot. In 10th International Workshop on Robot Motion and Control, RoMoCo, pp. 240-245. 2015. DOI:10.1109/RoMoCo.2015.7219742. 